\title{
UN BREVE COMENTARIO SOBRE LA DISFUNCIÓN DE LA ARTICULACIÓN SACROILÍACA
}

\section{A BRIEF COMMENT ON THE DYSFUNCTION OF THE SACROILIAC JOINT}

Marcos ALMIRÓN ${ }^{1}$.

${ }^{1}$ Kinesiólogo residente, Facultad de Ciencias Médicas, Universidad Nacional de Asunción, San Lorenzo - Paraguay.

Cómo citar este artículo: Almirón M. Un breve comentario sobre la disfunción de la articulación sacroilíaca. Medicina Clínica y Social. 2017;1(2):150-156.

\section{RESUMEN}

La articulación sacroilíaca es una estructura anatómica dentro del complejo lumbar, cuya disfunción está asociada a dolor de espalda baja. Una vez establecido el diagnóstico de disfunción de la articulación sacroilíaca, un tratamiento específico puede producir resultados satisfactorios. En este comentario se presentan brevemente aspectos relativos al examen físico y al abordaje terapéutico de pacientes con este diagnóstico.

Palabras clave: Articulación sacroilíaca; Dolor de espalda baja; Examen físico; Tratamiento.

\section{ABSTRACT}

The sacroiliac joint is an anatomical structure within the lumbar complex. Its dysfunction is associated with low back pain. Once the diagnosis of sacroiliac joint dysfunction has been established, a specific treatment can produce satisfactory results. This comment briefly presents aspects related to the physical examination and treatment approach of patients with this diagnosis.

Keywords: Sacroiliac joint; Low back pain; Physical examination; Treatment.

\section{RECUERDO ANATÓMICO Y FUNCIONAL}

La sacroilíaca es una articulación que une el sacro a la pelvis. En ella, el cartílago hialino del lado sacro se mueve contra el fibrocartílago del lado ilíaco. Por lo general, tiene forma de " $C$ ", con dos brazos de palanca que se entrelazan en el segundo nivel del sacro. Sus numerosas crestas y depresiones indican una función predominante de estabilidad, más que de movimiento; no obstante, se ha documentado que el movimiento si se produce en esta articulación, por lo que se podrían generar posiciones ligeramente subluxadas e incluso bloqueadas $(1,2)$. La articulación sacroilíaca debe su estabilidad a las crestas que posee y a la presencia de ligamentos. Éstos ofrecen resistencia al esfuerzo y a la carga, siendo los ligamentos profundos (anterior, posterior e interóseo) quiénes resisten la carga del sacro con relación al ilion. Los ligamentos superficiales (por ejemplo, los sacrotuberosos) reaccionan a movimientos dinámicos (elevación de la pierna recta, durante el movimiento) y el ligamento sacroilíaco dorsal se estira en periodos de lordosis lumbar (por ejemplo, durante el embarazo) $(3,4)$. 
Una rica cohorte muscular se relaciona con estos ligamentos y con la articulación sacroilíaca: el piriforme, el bíceps femoral, el glúteo mayor y el menor, el erector externo, el dorsal ancho, la fascia toracolumbar y el ilíaco. Cualquiera de los anteriores puede estar involucrado en un proceso doloroso a nivel de la articulación. La articulación, además, está ricamente inervada (5) por una combinación de terminaciones nerviosas libres no mielinizadas y de ramas primarias posteriores de L2-S3. Esta rica inervación explica por qué el dolor de la articulación puede manifestarse de muchas maneras, con patrones de referencia diferentes y únicos para cada paciente.

La función de la articulación es disipar cargas del torso, a través de la pelvis, hasta las extremidades inferiores y viceversa. La pelvis actúa como una base central a través de la cual se reciben y disipan grandes fuerzas. Aunque el papel principal de la articulación sacroilíaca es proporcionar estabilidad, la misma tiene movimiento limitado que le permite disipar y transferir cargas y tensiones significativas.

\section{EPIDEMIOLOGÍA}

La prevalencia de la disfunción de la articulación sacroilíaca se estima a través del estudio del dolor que produce. En ese sentido, investigaciones recientes han puesto de manifiesto que la prevalencia de dolor sacroilíaco oscila, según las series, entre el $13 \%$ y $30 \%$, de pacientes con dolor lumbar bajo $(5,6)$.

\section{ANAMNESIS Y EXAMEN FÍSICO}

Si bien el origen de una disfunción sacroilíaca puede deberse a causas mecánicas, inflamatorias, infecciosas o degenerativas, más de la mitad de los pacientes reporta que el inicio de la sintomatología se dio tras un evento injuriante (7). Factores de riesgo reconocidos por su asociación con el dolor de espalada baja ( $y$, también, con lesiones de discos lumbares) son el hábito tabáquico, una pobre condición física, historia familiar positiva, y labores relacionadas con el levantamiento de objetos. El embarazo puede ser considerado como una condición particular asociada a disfunción de la articulación sacroilíaca.

El elemento clave en el diagnóstico de disfunción sacroilíaca es el dolor. Éste por lo general se siente en uno o ambos glúteos y/o en o cerca de la espina ilíaca posterosuperior; no obstante, algunos pacientes reportan dolor que irradia a la cadera, al muslo posterior o incluso por debajo de la fosa poplítea. El dolor posee la cualidad de empeorar luego de que el paciente haya estado sentado durante largos periodos de tiempo o luego de realizar giros o movimientos rotatorios (7).

La tabla 1 informa de algunas características del dolor sacroilíaco (7).

TABLA 1. CARACTERÍSTICAS DEL DOLOR SACROILÍACO

\begin{tabular}{|c|l|}
\hline Calidad & $\begin{array}{l}\text { El dolor puede ser sordo o agudo. Algunos pacientes describen el dolor } \\
\text { como “provocado por un cuchillo". }\end{array}$ \\
\hline Distribución & $\begin{array}{l}\text { Las distribuciones más reportadas son las nalgas, la parte posterior del } \\
\text { muslo y la parte inferior de la espalda. El compromiso puede ser uni o bila- } \\
\text { teral. }\end{array}$ \\
\hline
\end{tabular}


Antecedentes inflamatorios o sistémicos
Un dolor que es peor durante las mañanas (rigidez matutina) y que se resuelve con el ejercicio es indicativo de una enfermedad inflamatoria. Asimismo, historia de fiebre, pérdida de peso y dolor con sudores nocturnos son posibles elementos de una enfermedad sistémica.

La tabla 2 indica los aspectos relevantes del examen físico de pacientes con disfunción de la articulación sacroilíaca.

TABLA 2. EXAMEN FÍSICO EN PACIENTES CON DISFUNCIÓN SACROILÍACA

\begin{tabular}{|c|l|}
\hline \multirow{2}{*}{ Inspección } & $\begin{array}{l}\text { La pelvis se presenta a menudo con altura asimétrica (lo que puede indicar } \\
\text { restricción unilateral de movimiento de una o ambas articulaciones). A tra- } \\
\text { vés de la prueba de flexión de cadera de Gillet se compara la simetría del } \\
\text { movimiento entre la espina ilíaca posterosuperior del lado evaluado y el } \\
\text { proceso espinal S2 (8). Se deben medir las longitudes de los miembros para } \\
\text { buscar desigualdad, inspeccionar la columna lumbar para buscar escoliosis } \\
\text { y girar las caderas para buscar restricciones de movimiento. }\end{array}$ \\
\hline Palpación & \begin{tabular}{|l} 
El dolor sacroilíaco puede despertarse con la palpación. El paciente suele \\
indicar de manera muy precisa, a través de un dedo, el punto doloroso en \\
particular (generalmente, el sulcus sacro, al lado de la espina ilíaca postero- \\
superior). Si el dolor es más difuso en la espalda, nalgas o piernas, se debería \\
cuestionar el diagnóstico de disfunción sacroilíaca (9).
\end{tabular} \\
\hline Examen neurológico & $\begin{array}{l}\text { En el examen neurológico, la fuerza muscular, la sensibilidad y los reflejos } \\
\text { en las extremidades inferiores están por lo general conservados. No obs- } \\
\text { tante, se debe recordar que a veces el paciente puede presentar debilidad } \\
\text { debido a la inhibición provocada por dolor o por desequilibrio franco del } \\
\text { músculo, desarrollado durante los episodios dolorosos o como consecuen- } \\
\text { cia de la inactividad. }\end{array}$ \\
\hline
\end{tabular}

Asimismo, las pruebas de provocación de dolor pueden ser utilizadas para detectar anomalías musculoesqueléticas e inflamación de las vértebras lumbares y la articulación sacroilíaca. Estas pruebas son:

- Distracción;

- Empuje del muslo;

- Flexión, abducción, rotación externa (FABER, por sus siglas en inglés);

- Compresión; y,

- Gaenslen.

Para obtener un resultado positivo en cualquiera de estas pruebas, se debe reproducir el dolor del paciente en la región de la articulación sacroilíaca. Si bien 1 prueba positiva genera sospechas, 3 o más pruebas positivas indicarían que la articulación sacroilíaca es la responsable de generar el dolor $(10,11,12)$. Una breve descripción de cada una de estas pruebas puede leerse en la tabla 3. 
TABLA 3. PRUEBAS DE PROVOCACIÓN DE DOLOR

\begin{tabular}{|c|c|}
\hline Distracción & $\begin{array}{l}\text { El paciente se encuentra en decúbito supino y el examinador aplica una } \\
\text { fuerza dirigida verticalmente hacia las espinas ilíacas anterosuperiores. Esto } \\
\text { genera una distracción de la cara anterior de la articulación sacroilíaca. La } \\
\text { prueba se considera positiva si reproduce los síntomas del paciente. Esto } \\
\text { indicaría disfunción sacroilíaca o esguince de los ligamentos sacroilíacos an- } \\
\text { teriores (13). }\end{array}$ \\
\hline Empuje del muslo & $\begin{array}{l}\text { Con el paciente en decúbito supino, la cadera se flexiona a } 90^{\circ} \text { (con la rodilla } \\
\text { doblada) para estirar las estructuras posteriores. Mediante la aplicación de } \\
\text { presión axial a lo largo de la longitud del fémur, éste se utiliza como palanca } \\
\text { para empujar el ilion posterior. Una mano se coloca debajo del sacro para } \\
\text { fijar su posición mientras que la otra mano se utiliza para aplicar una fuerza } \\
\text { hacia abajo al fémur (14). La prueba es positiva para el dolor de la cintura } \\
\text { pélvica si la presión axial provoca dolor sobre la articulación sacroilíaca. }\end{array}$ \\
\hline $\begin{array}{l}\text { Flexión, abducción y rota- } \\
\text { ción externa }\end{array}$ & $\begin{array}{l}\text { El paciente se coloca en decúbito supino. La pierna se coloca en flexión de } \\
\text { la cadera y abducción con el tobillo lateral (maléolo) descansando sobre el } \\
\text { muslo contralateral justo por encima (proximal a) de la rodilla). Mientras se } \\
\text { estabiliza el lado opuesto de la pelvis en la espina ilíaca anterior superior, } \\
\text { se aplica una rotación externa, una abducción y una fuerza dirigida poste- } \\
\text { rior, ligeramente aplicada a la rodilla ipsilateral hasta que se alcanza el } \\
\text { rango final de movimiento. Una prueba positiva es aquella que reproduce } \\
\text { el dolor del paciente o limita su rango final de movimiento (15). }\end{array}$ \\
\hline Compresión & $\begin{array}{l}\text { Con el paciente en decúbito prono, el examinador aplica una presión ante- } \\
\text { riormente dirigida sobre el sacro. Una mano se coloca directamente sobre } \\
\text { el sacro y se refuerza con la otra mano. El propósito es aplicar una fuerza de } \\
\text { cizallamiento anterior a ambas articulaciones sacroilíacas. La prueba es po- } \\
\text { sitiva si el dolor se reproduce en la región sacroilíaca (14). }\end{array}$ \\
\hline Gaenslen & $\begin{array}{l}\text { Con una cadera flexionada sobre el abdomen, se permite que la otra pierna } \\
\text { salga del borde de la cama. La presión debe ser dirigida hacia abajo en la } \\
\text { pierna para lograr la extensión de la cadera y estresar la articulación sacroi- } \\
\text { líaca. Si se produce dolor en el paciente, la prueba se considera positiva para } \\
\text { una lesión de la articulación sacroilíaca, patología de cadera, inestabilidad } \\
\text { de la sínfisis pubiana o lesión de raíz nerviosa }(10,11) \text {. }\end{array}$ \\
\hline
\end{tabular}

\section{IMÁGENES}

El examen imagenológico de la articulación sacroilíaca se realiza mediante radiografía anteroposterior de pelvis/columna lumbar. Las vistas sacroilíacas específicas superponen los márgenes articulares anterior y posterior, lo que puede aumentar la sensibilidad para detectar anomalías. Las radiografías así como la tomografía computada son eficaces para valorar la normalidad (o no) de la articulación. A través de la radiografía simple se pueden observar la presencia de divertículos, mientras que con la tomografía computada se puede hacer lo mismo para el examen de lágrimas anteriores, extravasación y comunicaciones a otras estructuras $(5,16)$. 


\section{TRATAMIENTO KINESIOLÓGICO-FISIOTERAPÉUTICO}

La fisioterapia se centra en el control del dolor y en la recuperación funcional. Modalidades como la radiación laser de baja frecuencia y tratamientos termoterápicos (calor o frío superficiales) pueden reducir el dolor. Terapias neurales como el masaje transverso profundo, la liberación miofascial y las técnicas de estiramiento muscular también pueden ayudar. El estiramiento miofascial pélvico, en posición neutra de la columna vertebral, puede usarse para alivio inmediato y a corto plazo del malestar. Asimismo, la movilización pasiva de la articulación sacroilíaca o la kinesioterapia propioceptiva (a través, por ejemplo, del método Kabat) pueden ayudar a la recuperación funcional. El fisioterapeuta identificará con el paciente aquellas actividades que agravan la condición, a fin de evitarlas (7).

En la fase de recuperación, la terapia física debe enfocarse en corregir cualquier asimetría mecánica o de longitud de miembros, estiramiento de músculos lumbopelvianos excesivamente tensos y fortalecimiento de músculos débiles/inhibidos. Lo anterior se realizará en posición neutra de la columna, a fin de minimizar el malestar. Por su parte, los ejercicios de estabilización se realizarán con el paciente en posición más dinámica y funcional, e incluirán actividades de equilibrio y propiocepción. Después de que el dolor del paciente se resuelva, y el mismo haya ya recuperado suficiente fuerza, la terapia de mantenimiento puede ser realizada en un gimnasio, previa guía realizada por el fisioterapeuta acerca de qué ejercicios realizar y con cuánta frecuencia.

\section{INTERVENCIONES MÉDICAS Y QUIRÚRGICAS}

Como en la mayoría de las condiciones que implican dolor musculoesquelético, muchos fármacos orales pueden proporcionar alivio inicial del dolor. Los antiinflamatorios no esteroideos son un pilar clave de la terapéutica y pueden combinarse con paracetamol para un efecto adyuvante. Cuando la lesión es aguda y asociado con espasmo muscular secundario, relajantes musculares o benzodiazepinas son opciones razonables. Sin embargo, estos medicamentos deben ser administrados con precaución y sólo para la fase aguda, inicial, del dolor, por el riesgo de tolerancia y dependencia que poseen. En casos crónicos, y principalmente cuando al dolor se le han añadido componentes psicológicos (por ejemplo, cognitivo-conductuales), se pueden utilizar antidepresivos y agentes anticonvulsivantes, que se han evidenciado eficaces en el tratamiento del dolor crónico y neuropático (17).

Si el dolor no se resuelve bien en las primeras 2-3 semanas, debe considerarse una inyección intra-articular bajo guía fluoroscópica. Por lo general, esta inyección comprende una mezcla de anestésicos y esteroides (18-20). La disminución del dolor post-inyección puede servir, también, para ofrecer información diagnóstica clara, cuando la fuente del dolor no está del todo dilucidada.

Finalmente, la intervención quirúrgica se utiliza raramente para el dolor no traumático de la articulación sacroilíaca (21). La cirugía se considera en pacientes con dolor crónico que ha durado años, con pobre calidad de vida, y que no han tenido éxito con el tratamiento kinesiológico y/o médico. 


\section{PRONÓSTICO}

La lesión sacroilíaca tiene, por lo general, un pronóstico de recuperación completa muy buena, siempre que se realice un diagnóstico precoz y tratamiento oportuno. Aunque la mayoría de los estudios sugieren que el $80 \%$ de las personas con una lesión en la parte inferior de la espalda mejoran significativamente dentro de las 2 semanas, aún hace falta realizar estratificaciones por grupos diagnósticos (7).

\section{CONFLICTOS DE INTERÉS Y FUENTE DE FINANCIACIÓN}

El autor declara no poseer conflictos de interés. Fuente de financiación: ninguna.

\section{REFERENCIAS BIBLIOGRÁFICAS}

1. Goldwaith JH, Osgood RB. A consideration of the pelvic articulations from an anatomical pathological and clinical standpoint. Boston Med Surg J. 1905;152(21):593-601. http://.dx.doi.org/10.1056/NEJM190506011522204

2. Edge-Hughes L. Hip and sacroiliac disease: selected disorders and their management with physical therapy. Clin Tech Small Anim Pract. 2007;22(4):183-194. http://.dx.doi.org/10.1053/j.ctsap.2007.09.007

3. Foley BS, Buschbacher RM. Sacroiliac joint pain: anatomy, biomechanics, diagnosis, and treatment. Am J Phys Med Rehabil. 2006;85(12):997-1006. http://.dx.doi.org/10.1097/01.phm.0000247633.68694.c1

4. Hungerford B, Gilleard W, Hodges P. Evidence of altered lumbopelvic muscle recruitment in the presence of sacroiliac joint pain. Spine. 2003; 28(14):1593-1600. URL.

5. Mejía G, Arias M, Valdez K, Carrillo S, Infante G. Dolor de la articulación sacroilíaca. Anatomía, Diagnóstico y Tratamiento. Rev. Soc. Esp. Dolor 2008;3:170-180. URL.

6. Bernard TN Jr, Kirkaldy-Willis WH. Recognizing specific characteristics of nonspecific low back pain. Clin Orthop Relat Res. 1987;217:266-280. URL.

7. Sherman AL, Gotlin R. Sacroiliac Joint Injury [Internet]. New York: Medscape; 2016 Nov 14 [cited 2017 Jul 07]. Available from: http://emedicine.medscape.com/article/96054overview

8. Freburger JK, Riddle DL. Using published evidence to guide the examination of the sacroiliac joint region. Phys Ther. 2001;81(5):1135-1143. URL.

9. Fortin JD, Falco FJ. The Fortin finger test: an indicator of sacroiliac pain. Am J Orthop (Belle Mead NJ). 1997;26(7):477-480. URL.

10. Gaenslen FJ. Sacro-iliac arthrodesis: indications, author's technic and end-results. JAMA.1927;89(24):2031-2035. http://.dx.doi.org/10.1001/jama.1927.02690240023008

11. Laslett M, Aprill C, McDonald B, Young S. Diagnosis of Sacroiliac Joint Pain: Validity of individual provocation tests and composites of tests. Man Ther. 2005;10(3):207-218. http://.dx.doi.org/10.1016/j.math.2005.01.003

12. Laslett M. Pain provocation tests for diagnosis of sacroiliac joint pain. Aust J Physiother. 2006;52(3):229. URL.

13. Cook C, Hegedus E. Orthopedic Physical Examination Test: An Evidence Based Approach. New Jersey: Prentice Hall; 2013.

14. Vercellini P. Chronic pelvic pain. London: Wiley-Blackwell; 2011. 
15. Flynn T, Cleland J, Whitman J. User's guide to the musculoskeletal examination: Fundamentals for the evidence-based clinician. Buckner, Kentucky: Evidence in Motion; 2008.

16. Fortin JD, Vilensky JA, Merkel GJ. Can the Sacroiliac Joint Cause Sciatica?. Pain Physician. 2003;6:269-271. URL.

17. Torales J, Arce A, Zacarías M, Girala N, Moreno M, Szwako A, et al. La Guía TAZ de Psicofarmacología Clínica. 1a ed. Asunción: EFACIM; 2014.

18. Manchikanti L, Abdi S, Atluri S, Benyamin RM, Boswell MV, Buenaventura RM, et al. An Update of Comprehensive Evidence-Based Guidelines for Interventional Techniques in Chronic Spinal Pain. Part II: Guidance and Recommendations. Pain Physician. 2013;16(2 Suppl):S49-S283. URL.

19. Friedly J, Chan L, Deyo R. Increases in lumbosacral injections in the Medicare population: 1994 to 2001. Spine. 2007;32(16):1754-1760. URL.

20. Murakami E, Tanaka Y, Aizawa T, Ishizuka M, Kokubun S. Effect of periarticular and intraarticular lidocaine injections for sacroiliac joint pain: prospective comparative study. J Orthop Sci. 2007;12(3):274-80. http://.dx.doi.org/10.1007/s00776-007-1126$\underline{1}$

21. Polly DW, Swofford J, Whang PG, Frank CJ, Glaser JA, Limoni RP, et al. Two-Year Outcomes from a Randomized Controlled Trial of Minimally Invasive Sacroiliac Joint Fusion vs. Non-Surgical Management for Sacroiliac Joint Dysfunction. Int J Spine Surg. 2016;10:28. http://dx.doi.org/10.14444/3028 